Cahiers d'études africaines

198-199-200|2010

50 ans

\title{
Intersections et trajectoires
}

Les études francophones et la théorie postcoloniale

Intersections and Trajectories. Francophone Studies and Postcolonial Theory

\section{Dominic Thomas}

\section{OpenEdition}

\section{Journals}

Édition électronique

URL : https://journals.openedition.org/etudesafricaines/16383

DOI : 10.4000/etudesafricaines. 16383

ISSN : 1777-5353

Éditeur

Éditions de l'EHESS

Édition imprimée

Date de publication : 20 novembre 2010

Pagination : 823-841

ISBN : 978-2-7132-2252-8

ISSN : 0008-0055

Référence électronique

Dominic Thomas, «Intersections et trajectoires », Cahiers d'études africaines [En ligne], 198-199-200 |

2010, mis en ligne le 02 janvier 2013, consulté le 23 avril 2022. URL : http://journals.openedition.org/ etudesafricaines/16383 ; DOI : https://doi.org/10.4000/etudesafricaines.16383

Ce document a été généré automatiquement le 23 avril 2022.

(c) Cahiers d'Études africaines 


\title{
Intersections et trajectoires
}

\author{
Les études francophones et la théorie postcoloniale \\ Intersections and Trajectories. Francophone Studies and Postcolonial Theory
}

\section{Dominic Thomas}

1 «Commençons par le commencement, ce qui est un bon point de départ » (Behr 2000: 3). C'est avec ce clin d'œil facétieux à la célèbre comédie musicale The Sound of Music que s'ouvre Embrace, le second roman de Mark Behr. De même que les enfants du film en question avaient été protégés des atrocités de la Seconde Guerre mondiale qui fournit le substrat textuel, il en va de même pour le jeune protagoniste du roman de Behr tandis qu'il fait son chemin à travers ce qui constitue un fascinant et provocateur paysage de complicité et de collaboration dans le contexte de l'apartheid sud-africaine. Dans les deux cas, une lecture heuristique dévoile un récit troublant semblable à celui rencontré dans l'examen de la relation entre les études francophones et la théorie postcoloniale elle-même. Le projet violent et déshumanisant que constituait le colonialisme a généré un corpus de textes africains ancrés principalement dans le domaine linguistique des traditions culturelles européennes, donnant naissance, au cours des années suivantes, à des modèles théoriques ayant essayé d'explorer et d'expliquer les circonstances coloniales et postcoloniales. Par conséquent, pour pouvoir juxtaposer les études francophones et postcoloniales, retracer leurs trajectoires autonomes, et délimiter leurs points d'intersection, il demeure essentiel de prendre en considération les histoires transcoloniales imbriquées, et cependant différentes, dans lesquelles une pléthore d'apparatus théoriques ont été déployés.

2 Les programmes que je propose consisteront à interroger l'histoire longue et compliquée de la France avec l'Afrique, à mettre en relief les composantes et contributions africaines aux études francophones, à souligner l'importance des modèles théoriques africains et à explorer et remettre en question la manière dont les études francophones et postcoloniales ont partagé, contesté, et même joui d'un terrain discursif de proximité sous l'égide d'une vaste gamme d'alignements disciplinaires aux États-Unis, de manière à retracer les nouvelles coordonnées et trajectoires intellectuelles de ces domaines et de façon à évaluer leur utilité et leur pertinence dans le processus de déballage des divers phénomènes culturels. 


\section{La rencontre avec la modernité}

3 L'expansionnisme colonial français était fondé sur l'adhésion au concept de modernité. Le progrès était par conséquent perçu comme une force de gravité vers l'assimilation d'un groupe spécifique d'idéaux universels qui ont servi d'indices à ce mouvement. Comme l'a démontré Bill Ashcroft (2001: 210) dans son ouvrage Post-Colonial Transformation, «la modernité était vue comme une période distincte et supérieure dans l'histoire de l'humanité [...] et "l'entrée" dans la modernité devenait le droit et l'obligation des puissances européennes ». Si, en effet, nous voulons bien admettre que le colonialisme, en tant que projet financé par l'État et mis en place à la suite de la Conférence de Berlin de 1884-1885, a fourni la «rencontre» discursive avec la modernité, alors on peut soutenir que les réactions africaines engendrées par ce monument historique doivent être comprises comme ayant été conçues et élaborées comme des tentatives pour contrer et démystifier les tenants et aboutissants fondateurs de ce projet². À cette fin, le travail et les activités de personnalités aussi importantes qu'Alioune Diop (fondateur de la revue Présence Africaine en 1947), Cheikh Anta Diop, Léopold Sédar Senghor et Albert Memmi deviennent centraux à toute considération des études francophones et postcoloniales, étant donné les diverses manières dont ils ont pris le contre-pied de la suprématie et de l'hégémonie intellectuelles occidentales en remettant en contexte et en soulignant les contributions africaines à l'art, la philosophie et la science. Car, alors que certains textes de Cheikh Anta Diop - principalement Nations nègres et cultures (1954)-et de Senghor - essentiellement les diverses anthologies de poésie disponibles actuellementcontinuent à recevoir une attention de la part des critiques, d'autres sont ignorés, ou tout au moins insuffisamment exploités dans les programmes scolaires ou négligés dans les recherches universitaires - notamment L'Afrique noire précoloniale (Diop 1960a), L'Unité culturelle de l'Afrique noire (ibid. 1960b), Antériorité des civilisations nègres (ibid. 1960c) et Civilisation ou Barbarie (ibid. 1981), ainsi que la série Liberté I-IV de Senghor (1964, 1971, 1977, 1983). Cela devient tout particulièrement évident lorsque l'on considère le corpus remarquable de recherches consacrées par les Antillais au contenu et à la forme de l'expérience caribéenne - Aimé Césaire (1955), Frantz Fanon (1952), Édouard Glissant (1981) et Jean Barnabé, Raphäl Confiant et Patrick Chamoiseau (1989). En effet, les études francophones et postcoloniales pourraient accroître considérablement leur validité et leur authenticité en continuant à insister sur le besoin d'un modèle qui soit aussi démocratique et inclusif que possible en ce qui concerne les diverses zones géographiques au sein desquelles la pensée est générée. Les populations de l'Afrique subsaharienne continuent à nourrir un ressentiment transgénérationnel envers le discours français réducteur au sujet de l'Afrique. En sousestimer la portée revient à profondément méconnaître la relation ténue et complexe entre les anciennes colonies francophones de l'Afrique subsaharienne et la métropole.

4 Cet article se situe donc principalement dans un contexte " francophone ", et le terme lui-même sert naturellement à souligner les connexions et interconnexions historiques entre l'Hexagone et des régions du monde qui furent autrefois colonisées ou qui demeurent occupées - tels les départements d'Outre-mer (Dом) et les territoires d'Outre-mer (том) - et qui continuent à être soumis aux pratiques hégémoniques françaises sous le terme de "francophonie » (bien sûr, l'ironie des Antilles françaises comme faisant «intégralement» partie de la France doit ici être soulignée). De 
plusieurs façons, le lien symbiotique entre la littérature française et la littérature francophone d'Afrique subsaharienne trouve son origine dans le discours français sur l'Afrique dans plusieurs textes et traditions littéraires, tels que le projet de l'Encyclopédie au XvIII ${ }^{\mathrm{e}}$ siècle, les travaux d'Arthur Gobineau, Prosper Mérimée et de l'abbé Grégoire, parmi d'autres, et les représentations qui ont émergé de cette pensée. En effet, on pourrait soutenir l'argument selon lequel ces représentations ont fourni une grande partie des justifications de l'expansionnisme colonial, une activité missionnaire accrue, des pratiques assimilationnistes et, bien sûr, la mission civilisatrice en tant que projet idéologique. L'expansionnisme colonial et impérial a fourni les exemples historiques parmi les plus frappants des impulsions totalitaires des tendances universalisantes, notamment l'imposition de normes dans les communautés que les puissances coloniales étaient collectivement et sans discrimination engagées à briser. Si l'on comprend l'« assimilation " comme étant un processus de fusionnement, alors la notion de fusionnement n'est pas appropriée dans le contexte colonial, dans la mesure où les mécanismes de colonisation s'investissaient dans la réduction de l'autre vers le " même »-c'est-à-dire s'employaient à forger des prototypes culturels qui adhèrent aux idéologies coloniales. La logique derrière cette fusion d'éléments culturels contient l'implication que le "métissage» serait en théorie possible, dimension qui serait contraire aux idéaux de l'«assimilation» française, étant donné que la rhétorique justifiant l'expansionnisme était fournie par l'objectif de l'impératif «civilisateur » de compenser ce qui était perçu comme les faiblesses culturelles, linguistiques, politiques, religieuses et sociales du colonisé.

5 Les mécanismes coloniaux français furent érigés sur un paradigme assimilationniste ethnocentrique refusant d'interpréter la culture comme processus dynamique et, par conséquent, d'incorporer les éléments culturels africains. Au lieu de cela, les actions françaises elles-mêmes servent de témoignage des efforts dirigés vers le rejet, la répudiation, le démantèlement et l'effacement systématique des contributions africaines à une sorte d'entité universelle. Le traitement du multiculturalisme en France et de la postcolonie, et, à leur tour, les différentes façons dont les études françaises et francophones ont exploré la cohabitation dans les universités américaines, reflètent cette histoire coloniale. Je reviendrai sur cette question plus loin dans cet article. Dans une certaine mesure, il s'agit là d'une perspective développée par Emily Apter dans son essai «French Colonial Studies and Postcolonial Theory » (1995: 169) dans lequel elle signale que « de nombreux intellectuels français semblent avoir du mal à comprendre la pertinence de la théorie postcoloniale pour la politique contemporaine de la culture».

6 Au sein de la structure de cet article, je propose d'aller un peu plus loin et d'établir une distinction entre les problèmes culturels et économiques dans les contextes de la globalisation. Ashcroft (2001: 208) a qualifié les tendances récentes de « recolonisation", mais a insisté sur le fait que nous reconnaissions que, bien que le Fonds monétaire international (FMI), la Banque Mondiale et les entreprises multinationales «aient une lourde influence sur la politique intérieure des pays » (ibid. : 209), une distinction importante doit être faite, puisque ces organisations ne sont pas impérialistes en ce qu'elles n'opèrent pas comme des états autonomes (ici, on peut remarquer une transition importante depuis l'influence occidentale coloniale et néocoloniale). Néanmoins, divers praticiens de la culture ont consacré leur travail à la dénonciation de ces récentes tendances comme étant de simples réarticulations et reconfigurations d'anciennes traditions d'exploitation - le film Hyènes (1992) du 
cinéaste sénégalais Djibril Mambéty Diop et le documentaire de Glen Ellis dénonçant les activités de la société Shell au Nigeria, The Drilling Fields (1994) fournissent des exemples frappants de l'orientation prise par les travaux récents dans leurs efforts de démontrer comment ces organisations fonctionnent de la même manière que des États, n'ayant aucun compte à rendre de leur comportement, employant souvent les services de milices privées, et insistant sur le fait que leurs activités sont bénéfiques aux populations indigènes pour qu'elles puissent participer à des modes d'existence fondés sur la modernité.

7 Ashcroft (2001:214) a fourni un résumé des deux approches principales qui informent la définition de la globalisation: "D'une part, la culture est vue comme étant essentiellement territoriale, la possession d'une société ou d'un groupe social particuliers; d'autre part, elle est vue comme un faisceau malléable de stratégies de négociation de la réalité d'un groupe, un processus d'apprentissage translocal. Selon ces positions, la culture est soit [...] formée par des sociétés et nations, soit transformée par des diasporas et l'intercommunication. » Ainsi, pour Ashcroft, la mondialisation est soit homogénéisante/hégémonique, soit hétérogène/interpénétrante (ibid.). Si la mondialisation est, en effet, un phénomène "interpénétrant ", alors peut-être que le régional n'est en fait pas fermé et que la diaspora pourrait avoir un rôle à jouer dans la redéfinition du régional en dehors de ces tendances universalisantes ayant une dette envers l'Occident. Cependant, cela ne sera pas possible tant que nous ne nous serons pas distanciés des dangers inhérents à la reformulation de l'argument de la "modernité", en fonction des paramètres ténus du discours actuel qui fait de «l'acquis » et de « l'exploitation » des synonymes (ibid. : 212). De plus, l'insistance de la France à protéger "l'exception française " dans le domaine culturel est elle-même problématique dans son programme hégémonique anti-culturel, particulièrement quand on considère l'effort concerté fait par les Services culturels français aux ÉtatsUnis pour promouvoir la francophonie en tant qu'indice de la soi-disante ouverture de la France à la diversité culturelle. Le Sommet des chefs d'État francophones s'était initialement mis d'accord en 1986 pour que soient inclus parmi ses membres « ces pays ayant en commun l'usage du français ", et, tandis que le critère d'inclusion se déplaça plus tard vers «les pays partageant l'usage du français ", offrant ainsi peut-être un paradigme qui pourrait défier la légitimité de la formation du canon en décentralisant l'hégémonie linguistique de façon à générer un modèle plus inclusif, il faut insister sur le fait que ces mesures n'impliquent en rien que la domination métropolitaine de la francophonie ait cessé.

\section{L'Afrique au carrefour}

8 Comme l'a soutenu Christopher L. Miller (1990), «l'étude de la littérature noire africaine en français requiert une approche qui soit sensible à la fois aux différences ethniques locales et aux effets homogénéisants de la langue française. Il s'en suit que le rôle que doit jouer la "théorie" devrait être prudemment déterminé en se demandant dans quelle mesure elle est appropriée $»^{3}$. Les travaux des théoriciens les plus connus, Homi Bhabha, Edward Said et Gayatri Chakravorty Spivak (parmi tant d'autres, bien sûr) ont reçu l'attention qu'ils méritent de la part de la critique. Cependant, comme je l'ai mentionné auparavant, de nombreux auteurs africains demeurent insuffisamment lus en comparaison avec leurs homologues anglophones et antillais, ce qui rend nulle la possibilité de modèles véritablement inclusifs. Alors que Miller (ibid. : 25) insiste sur le 
besoin de « reconsidérer d'un œil sceptique l'applicabilité de tous les termes critiques occidentaux et de rechercher dans les cultures africaines traditionnelles quels termes elles ont à offrir ", la manière la plus efficace de réaliser cette fin est peut-être d'attirer l'attention des chercheurs sur les travaux de ces théoriciens africains qui sont réduits au silence par l'économie des mots - la distribution du savoir, l'économie de la publication, la circulation des ressources humaines dans le milieu universitaire «global».

9 À ma première lecture de l'ouvrage de Leila Gandhi, intitulé Postcolonial Theory (1998: 141), je fus quelque peu frappé par la définition qu'elle donne de la «théorie postcoloniale »: «Une catégorie contentieuse qui fait référence de façon quelque peu arbitraire aux "littératures en anglais", c'est-à-dire ces littératures qui ont accompagné le projet et le déclin de l'impérialisme britannique. » J'ai réfléchi initialement pendant quelque temps à la signification plus profonde de cette observation, rejetant d'abord sa véracité, situant les origines de sa formulation dans ce que je concevais comme étant l'absence de conscience, de la part de l'auteur, du vaste corpus de réactions critiques et de littérature généré dans les sites francophones, lusophones et autres sites linguistiques. Puis, revenant à ce que j'avais identifié comme étant peut-être le mot-clé dans son observation - «arbitraire » -, je commençai à penser qu'elle avait peut-être mis le doigt sur quelque chose. D'une part, que d'autres modes de recherche pouvaient en fait accomplir ce à quoi nous faisons référence comme étant la théorie postcoloniale sans utiliser ce terme et, d'autre part, qu'un alignement disciplinaire et institutionnel pouvait en fait entraver le débat et le dialogue à travers les différentes frontières réelles et imaginaires qui ont été érigées pour étouffer leurs qualités poreuses. Un survol sommaire des contributions les plus importantes à la théorie postcoloniale confirme en vérité que ce domaine discursif a été dominé presque exclusivement par des travaux produits en Australie, en Nouvelle-Zélande, en Inde, en Grande-Bretagne, au Canada et aux États-Unis au sein des facultés d'anglais, de lettres, d'études culturelles et de littérature comparée, se concentrant, par conséquent, presque exclusivement, sur des sources primaires ayant émergé des traditions culturelles de ces régions. Il existe bien sûr des exceptions qui comprennent des textes lus fréquemment de Ngùgì wa Thiong'o, Frantz Fanon, des textes sur la question de la négritude, et, dans une certaine mesure par Léopold Sédar Senghor. The Post-Colonial Studies Reader, édité par Bill Ashcroft, Gareth Griffiths et Helen Tiffin (1995), Colonial Discourse and PostColonial Theory, édité par Patrick Williams et Laura Chrisman (1994), Dangerous Liaisons, édité par Anne McClintock, Aamir Mufti et Ella Shohat (1997), ainsi que Postcolonial Theory par Bart Moore-Gilbert (1997), exécutent tous ce "geste $»^{4}$. Il est clair que des problèmes d'exclusivité et de perspective limitée sont en question ici, bien qu'il s'agisse plus du résultat d'une formation disciplinaire que d'une mise en accusation du programme guidant ces volumes - un tel « geste » serait en fait futile. En soulignant les contributions importantes que ces travaux apportent à la reconnaissance de productions culturelles riches et diversifiées, je m'efforce plutôt de souligner le fait que ce qui est exclu nous conduit parfois à la triste prise de conscience et reconnaissance que nous avons tous des collègues, qui, souvent, travaillent dans la même institution, desquels nous avons tant à apprendre en termes d'échanges collaboratifs.

La théorie postcoloniale n'a pas parlé suffisamment des travaux des théoriciens et écrivains africains. Cela est en grande partie dû aux barrières de la langue bien sûr, mais aussi, de manière plus significative, à la nature de la configuration des facultés 
dans les universités américaines, dont une analyse peut nous permettre d'avancer dans la compréhension des circonstances actuelles, ainsi que dans la détermination de la tâche à laquelle nous sommes confrontés afin de rectifier et corriger la situation. Un certain nombre d'universitaires ont déjà fait d'importantes incursions dans le redressement de ce déséquilibre. Dans le volume édité par Georg M. Gugelberger (1985), Marxism and African Literature, les contributions reflétaient une approche supranationale et multilingue (anglophone, francophone et lusophone), et des travaux par des auteurs venant de pays aussi divers que le Kenya, le Mozambique, l'Afrique du Sud, le Nigeria et le Mali y étaient examinés. L'ouvrage de Chidi Amuta (1989), The Theory of African Literature, adopte un schéma transcolonial afin d'explorer des textes africains de Cabral, Neto, Ngùgì et Sembène, tandis que Christopher L. Miller (1990), dans son ouvrage intitulé Theories of Africans, élargit considérablement le champ de recherche en insistant sur le fait que «la tâche consiste à rechercher une meilleure compréhension de la littérature africaine francophone en la replaçant dans son contexte historique, politique, mais surtout anthropologique ». À son tour, Françoise Lionnet (1995) a démontré le potentiel d'investigations comparatives et transnationales (Algérie, Botswana, Égypte, Guadeloupe, Jamaïque, Maurice) dans Postcolonial Representation. Plus récemment, des ouvrages par des théoriciens francophones très en vue travaillant à l'intersection de la littérature, de la philosophie, des sciences politiques et de la sociologie - notamment le remarquable ouvrage De la Postcolonie d'Achille Mbembe (2000) - ont commencé à être de plus en plus lus dans certains cas, seulement parce que leurs travaux sont devenus disponibles en traduction.

11 Mon objectif n'est pas de fournir une analyse exhaustive et approfondie des modèles théoriques africains les plus importants, mais plutôt d'identifier certains des paradigmes-clés qui ont émergé et qui, par implication, s'adressent à la théorie postcoloniale. Parmi ceux-ci, le projet de la négritude lui-même occupe une place proéminente, dont l'originalité est née de ce que Senghor (1977:69) a décrit comme la tentative de «l'élaborer comme arme de combat, instrument de libération à l'Humanisme du xxe siècle ». Comme je l'ai mentionné précédemment, le présupposé de la conscience identitaire a constitué l'une des premières étapes dans la lutte contre le discours hégémonique réducteur qui, à la fois, justifiait et demeurait une composante intrinsèque du projet colonial. Les travaux de Senghor lui-même, ainsi que ceux d'une pléthore de romanciers francophones d'Afrique subsaharienne, ont traité de ce sujet à travers leur considération de divers aspects du colonialisme et de son impact sur la culture, la philosophie, la psychologie et la religion africaines. Les articulations les plus convaincantes de ces questions ont d'abord été formulées par Camara Laye, Mongo beti, Ferdinand Oyono, Cheikh Hamidou Kane, Bernard Dadié, Tchicaya U Tam'si et Ousmane Sembène au cours des années 1950 et 1960. Elles ont plus tard été recontextualisées selon les circonstances politiques changeantes liées à la postcolonialité par Alioum Fantouré, Aminata Sow Fall, Massa Makan Diabaté, Ahmadou Kourouma, Henri Lopes, Emmanuel Dongala, Mariama Bâ et Sony Labou Tansi. Au carrefour du nouveau millénaire, ces questions ont de nouveau été soulevées dans la nouvelle génération de textes étonnamment originaux produits par Abdourahman Waberi, Eugène Ébodé, Nangala Camara, Daniel Biyaoula, Alain Mabanckou et Boris Boubacar Diop. Les réactions critiques soulignèrent les préoccupations de ces écrivains - organisées autour des problèmes de genre, d'influence littéraire, d'idéologie, d'historiographie, de littératures nationales, de postcolonialité, et, bien sûr, les discours générés par la 
situation d'exil et la supposition de résidence dans diverses communautés diasporiques africaines.

L'analyse d'Achille Mbembe (2000), dans son ouvrage De la Postcolonie, sert à souligner la manière dont l'Afrique et le discours occidental demeurent imbriqués. De nombreuses façons, ce cadre contextuel fait écho à ma propre position en ce qui concerne la manière dont, à la fois, les études francophones et la théorie postcoloniale et les études françaises et francophones demeurent nécessairement symbiotiques. Pour Mbembe, « il n'y a presque jamais de discours sur l'Afrique pour elle-même. Dans le principe même de sa constitution, dans son langage et dans ses finalités, l'énoncé sur l'Afrique est toujours le prétexte à un propos sur quelque chose d'autre, quelque autre lieu, d'autres gens. Plus précisément, l'Afrique est cette médiation grâce à laquelle l'Occident accède à son propre inconscient et rend publiquement compte de sa subjectivité " (Miller 1985). Ces observations nous conduisent donc à réfléchir plus amplement à la relation entre les études francophones, la théorie postcoloniale et les études africaines, tout en nous offrant également l'opportunité de suggérer diverses manières dont nous pourrions commencer à tracer la trajectoire des futurs alignements des programmes scolaires. En effet, cette direction a déjà été pensée, et mon argument constitue simplement une contribution supplémentaire à ce qui devrait faire l'objet d'un débat continu. Il est clair, cependant, que des discours alternatifs sont en train d'être créés avec certaines implications politiques et pédagogiques.

Quand on regarde l'histoire des facultés de français aux États-Unis - le profil et la formation des professeurs, les objectifs déclarés, l'orientation du programme -, on découvre que la configuration des facultés reflète, la plupart du temps, la structure de leurs homologues outre-Atlantique (en effet, c'était le cas en Grande-Bretagne également jusque dans les années 1980, situation mieux illustrée par le refus de certains professeurs des prestigieuses universités d'Oxford et de Cambridge d'enseigner des auteurs vivants - il existe en vérité des récits qui circulent au sujet d'étudiants célébrant la mort prématurée d'Albert Camus en 1960 !). Il existe bien sûr de nombreuses manières dont le contexte américain a offert aux universitaires une perspective et une distance par rapport aux divers textes étudiés, ce qui, en retour, a donné lieu à la richesse de la production universitaire provenant de chercheurs basés aux États-Unis - du structuralisme à la déconstruction, en passant par la queer theory, les gender studies, etc. Cette production universitaire, plutôt que d'avoir créé ce que j'ai déjà entendu décrire comme version lite des études françaises (pour mentionner le terme utilisé en France pour désigner les boissons diététiques), a, au lieu de cela, fourni au domaine la vitalité et le dynamisme qui ont maintenu son statut au premier plan des disciplines littéraires. Je partage la croyance fondamentale selon laquelle les facultés de français sont sur le point d'être transformées par de nouvelles approches et de nouveaux domaines de recherche, mais mes raisons pour atteindre cette conclusion diffèrent considérablement de la critique implicite contenue dans l'allusion au terme lite. La résistance aux études francophones (et, par association, à la théorie postcoloniale elle-même) dans les facultés de français s'aligne avec les objections que j'ai mentionnées ci-dessus à certaines conceptions de ce à quoi des études françaises devraient ressembler, mais, de façon plus significative, à un rejet des littératures produites en Afrique, au nord et au sud du Sahara, au Canada, aux Antilles, dans l'Océan Indien, au Proche-Orient, en Asie, dans la région du Pacifique, dans les pays européens ayant une tradition de langue française, et, bien évidemment, au sein des communautés postcoloniales en France. Cependant, la situation est en train de lentement changer, et 
la plupart des facultés de français (dont certaines ont même changé leur nom en faculté «d'Études françaises et francophones» afin de refléter cette orientation supplémentaire) possèdent maintenant au moins un professeur spécialisé en ce domaine. Néanmoins, étant donné les régions géographiques que ces spécialistes sont amenés à enseigner, la question demeure clairement de savoir si un seul spécialiste de la francophonie par faculté suffit. Ces positions sont parallèles à l'échec de la France à incorporer adéquatement les littératures du monde francophone dans leur cadre institutionnel, précisément à cause de leurs origines compliquées dans les histoires coloniales - contextes raciaux/ethniques et mentalités - dont la France doit venir à bout.

14 L'analyse de l'orientation des programmes et alignements universitaires dans les institutions américaines fournit une feuille de route intéressante pour un voyage dans le monde des études francophones et postcoloniales, et c'est avec une intention délibérée que j'utilise la notion "d'intersection» dans le titre de cet article afin de souligner cette relation. Car, si la conséquence du colonialisme européen et de l'expansionnisme impérial est l'émergence des nations africaines, comme le prouvent les alignements cartographiques actuels, alors les diverses influences linguistiques et les publications qui s'en suivent sont également le témoignage de cet héritage de l'influence occidentale. L'Afrique (et d'autres régions du monde non occidental) a été marginalisée dans le programme universitaire, de par sa relégation aux area studies, centres et programmes. L'interdisciplinarité a été étouffée dans les études françaises à cause de la nature fermée de ce domaine, et il n'est pas surprenant que des avancées plus significatives aient été faites dans de nombreuses facultés de littérature comparée qui - au moins en principe - se définissent par la structure intégrée qu'elles offrent pour l'exploration de phénomènes culturels au-delà des différentes frontières. Cependant, l'une des manières passionnantes dont nous pourrions établir des connexions entre les cultures anglophones, francophones et autres cultures postcoloniales, est précisément par le biais d'approches transcoloniales et transnationales, dans lesquelles la relation de ces domaines avec d'autres influences culturelles (arabes ou islamiques) pourrait être soulignée. Bien sûr, des structures interdisciplinaires plus larges sont, aujourd'hui, caractéristiques de la recherche la plus séduisante dans le domaine des études françaises, de la période médiévale à l'époque contemporaine.

Le passé colonial de la France, ainsi que l'histoire de la décolonisation, de l'immigration de l'après-guerre, et l'expansion de la communauté européenne (parmi toute une gamme d'autres facteurs socioculturels), ont bien sûr transformé la structure démographique, institutionnelle et politique de la France. Le volume de Sub-Stance, édité par Lawrence Kritzman (1995: 13), a remis en question le programme de Pierre Nora (1984-1992) dans les sept volumes de ses Lieux de mémoire, projet structuré comme " résultat d'un processus imaginaire qui codifie et condense une conscience nationale du passé ", par rapport à une mémoire collective qui jouit aujourd'hui de coordonnées radicalement différentes. En effet, un observateur étranger/extra-terrestre cultivé, ayant fraîchement atterri dans le désert du Nevada, pourrait penser que Pierre Nora a été commissionné pour concevoir Paris-Vegas, site architectural qui témoigne de la vision démodée et stéréotypée de ce à quoi correspond la France - la Tour Eiffel, l'Arc de Triomphe et le Louvre - et à quoi nous sommes régulièrement confrontés dans nos contacts avec les étudiants et dans les articles sur la France. De façon similaire, Achille Mbembe émet les observations suivantes au sujet de l'interférence continue de 
l'Occident (simultanément par l'exercice du pouvoir par des organisations gouvernementales et multinationales) dans le gouvernement des nations africaines souveraines. Dans ce contexte, un pays comme le Congo "est un exemple d'extraterritorialisation. Ici, le modèle n'est pas celui de la partition proprement dite, mais celui du tourbillon. Ces tourbillons sont cycliques et ont pour épicentre la capitale, Brazzaville. Située à l'intérieur du pays, celle-ci a son centre de gravité hors d'ellemême, dans la relation que l'État entretient avec les compagnies pétrolières opérant en haute mer" (Mbembe 1999: 22). Les observations de Mbembe pourraient servir d'exemple de la manière dont les études françaises pourraient commencer à se déterritorialiser en encourageant des innovations méthodologiques qui exerceraient une influence sur le futur de l'interdisciplinarité.

\section{L'économie des mots et le tracé de nouvelles routes}

16 Tout le monde sait aujourd'hui que Paris et, dans une moindre mesure, Londres, demeurent les centres de publications pour les écrivains originaires de territoires ayant des liens coloniaux avec la France et la Grande-Bretagne. La longue liste des auteurs qui, au cours des dernières années, ont été soit nominés soit lauréats des prix littéraires les plus convoités en France (le Goncourt et le Renaudot) et en Grande-Bretagne (le Booker) est impressionnante. Elle comprend Tahar Ben Jelloun et Patrick Chamoiseau, dans le cas du Goncourt (Aminata Sow Fall fut nominée en 1979), et Ahmadou Kourouma, Alain Mabanckou et Tierno Monénembo pour le Renaudot, tandis que le Booker fut décerné à J.M. Coetzee (à deux reprises!), Nadine Gordimer, Salman Rushdie, Ben Okri, et Arundhati Roy. À cette fin, la dimension ironique et controversée des paroles de Zadie Smith $(2001: 51,2003)$ semble d'autant plus pertinente: "Quel genre d'expression est-ce que? "So what?" Est-ce de l'anglais? Ce n'est pas de l'anglais. Seuls les immigrés peuvent parler l'anglais de la reine de nos jours.» Cet ouvrage extraordinaire, écrit par une jeune anglo-jamaïquaine qui a publié à l'âge de vingt-quatre ans cette œuvre maitresse, dont les protagonistes font face aux défis et paradoxes d'une Angleterre multiculturelle, dresse un portrait osé de la « Nouvelle Angleterre » avec humour et franchise. Le roman connut un énorme succès, donna lieu à de nombreux débats publics et fut adapté à la télévision en septembre 2002.

Néanmoins, ces tendances ont un aspect plus ambigu, voire sinistre. Le cas de la France en est peut-être le plus révélateur, dans la mesure où la plupart des auteurs africains francophones sont publiés en France. Les maisons d'édition spécialisées, comme Présence Africaine et L'Harmattan, publient la majeure partie de ces auteurs (en fait, une fois que leurs ouvrages ont atteint une certaine notoriété, les écrivains passent souvent à de plus grandes maisons d'édition - Henri Lopes a commencé avec CLÉ et Présence Africaine et est passé aux Éditions du Seuil en 1990 ; Alain Mabanckou a quitté Présence Africaine en 2002 pour le Serpent à plumes pour ensuite rejoindre également les Éditions du Seuil en 2006 et Gallimard en 2010), tandis que les plus grands éditeurs insistent sur le fait qu'ils ne sont capables de se concentrer que sur un nombre sélectionné d'écrivains «africains». Ce n'est que rarement que ces éditeurs promeuvent de nouvelles voix, et l'on fait très peu d'efforts pour établir et aider les maisons d'édition africaines indépendantes. L'une des plus grandes librairies de France, la FNAC, continue à opérer une ségrégation entre les auteurs écrivant en français selon les régions géographiques dont ils sont originaires - France, Maghreb, Afrique noire, Antilles, etc. -, tandis que l'une des plus importantes maisons d'édition, Gallimard, a 
récemment lancé une nouvelle collection, Continents noirs, fournissant ainsi l'un des exemples les plus flagrants de la récupération de la périphérie par le centre, maintenant de ce fait la hiérarchie et le statut quo. Cependant, paradoxalement, alors que l'on pourrait soutenir que ces efforts permettent au moins à certains écrivains d'être lus, une autre perspective pourrait suggérer que les institutions qui décernent les prix littéraires aient en vérité plus besoin de ces écrivains qu'inversement (récemment, ce débat fut à nouveau relancé suite à l'attribution du Prix Goncourt à la franco-sénégalaise, Marie NDiaye). En effet, Sony Labou Tansi (Orisha 1986) avait un jour déclaré : «Il faut dire que s'il y a du français et de moi quelqu'un qui soit en position de force, ce n'est pas le français, c'est moi. Je n'ai jamais eu recours au français, c'est lui qui a recours à moi. » Gardant cette réflexion à l'esprit, je propose d'ébaucher certaines des manières dont les études françaises aux États-Unis devraient insister sur un modèle plus inclusif afin d'assurer leur survie en tant que domaine d'étude.

Il ne s'agit pas d'un débat qui concerne la territorialité disciplinaire, mais plutôt l'expression de la position précaire du français dans les institutions américaines, ainsi qu'une tentative concertée d'articuler la façon dont on pourrait s'assurer de la perduration de la pertinence des études françaises dans les sciences humaines. Si elles ne réfléchissent pas sérieusement à cette question, les facultés de français courent le risque de devenir de simples fournisseurs de service pour d'autres instances sur le campus, et le terrain de formation pour l'acquisition d'une compétence linguistique professionnelle en dehors d'un contexte culturel. Les programmes devraient mieux refléter à la fois les réalités des missions institutionnelles $\mathrm{du} \mathrm{xxI}^{\mathrm{e}}$ siècle et les intérêts des étudiants - et cela est peut-être encore plus important - les besoins, exigences et réalités du marché de l'emploi pour les étudiants au-delà de l'université. Au lieu d'annoncer la mort des facultés de français, ces changements suggèrent de nouvelles façons de penser l'éducation, et de définir le rôle que le français pourrait, et devrait, jouer dans l'avenir. Tout en maintenant les taux d'inscription, l'intérêt des étudiants, et ainsi de suite, ces changements contiennent la promesse d'accroître l'intérêt pour l'étude des langues, ainsi que l'internationalisation de l'expérience estudiantine, tout en préparant simultanément mieux les étudiants pour l'éducation et la formation postuniversitaire.

19 L'une des manières de mettre en œuvre ce changement consiste à commencer par faire le point sur la présence des études françaises sur les campus en général. Plutôt que d'avoir constamment à justifier et établir des critères pour l'embauche de nouveaux professeurs, les facultés de langue " devraient " récupérer les cours pertinents offerts sur le campus qui pourraient devenir centraux à leur mission. Pourquoi, par exemple, les ouvrages des anthropologues, historiens, cinéastes, sociologues, artistes français, etc., devraient-ils rester le domaine privilégié d'autres facultés ? Pourquoi Gramsci estil enseigné en sociologie et non pas en italien? Pourquoi Marx est-il confiné à la philosophie et aux sciences politiques, et non pas également offert au sein des facultés d'allemand (en version originale et en traduction)? Le dialogue inter-faculté, l'enseignement en équipe et les cours offerts simultanément dans plusieurs facultés devraient être encouragés de toutes les manières possibles de façon à s'efforcer de créer les possibilités de véritable interdisciplinarité. Étant donné le contexte colonial de l'accession de la France à la centralité, cela ne devrait pas être perçu comme une tentative de colonisation de l'interdisciplinaire mais plutôt comme l'indice de la manière dont la défense d'une version traditionnellement conservatrice des études françaises résultera inévitablement dans sa disparition. Les facultés de langue ont 
besoin d'offrir ce genre de cours et d'être activement engagées dans le recrutement de professeurs ayant une formation et un doctorat dans des domaines liés. De plus, ces mesures ne seraient pas conçues pour éliminer d'autres unités institutionnelles, mais plutôt pour enrichir le travail fait au sein des diverses facultés et pratiques pédagogiques. Comme l'a soutenu Dominick LaCapra (2000: 193-194) dans son article «Reconfiguring French Studies »:

« Un troisième défi implique le mouvement ou l'expansion du champ d'intérêt de la littérature à la culture, à la société et à l'histoire, y compris les cultures, sociétés et histoires francophones. Une tentative de réarticulation et de recadrage du champ des études françaises est d'explorer de façon critique le problème ardu de l'interaction entre théorie, critique, historiographie, ethnographie, et l'étude de la littérature ou d'autres formes artistiques - problème qui pourrait être pris de manière trompeuse comme solution appelée études culturelles. »

20 J'adhère de tout cœur à l'insistance de LaCapra sur les approches plus vastes et, je suis d'accord sur le fait que « cette reproblématisation est [...] nécessaire si le domaine doit être vital et dans une position viable de se faire une place au sein d'une configuration de disciplines qui est en train d'être redéfinie face aux demandes à la fois intellectuelles et économiques » (ibid. : 226).

21 Ces réformes ne sont pas, de quelque manière que ce soit, conçues pour générer des échanges antagonistes ou promouvoir le conflit, mais plutôt pour souligner la nature mutuellement constituante d'approches différentes des phénomènes culturels. En reconnaissant et réagissant à la réalité de l'hégémonie croissante de la langue anglaise, il existe un besoin urgent de mettre en relief les nombreuses façons dont toutes les approches de la culture et de la littérature ont un bénéfice à tirer de la coopération, qu'il s'agisse des soi-disant approches traditionnelles ou bien de celles qui emploient des outils critiques plus récents. Ce n'est pas une question de gagnant ou de perdant, de programmes standardisés, lite, mais plutôt d'encourager un dialogue productif qui commence par l'acceptation du fait que la réinterprétation et la recontextualisation sont nécessaires. Comme l'a suggéré Apter (1995: 172), «la critique postcoloniale française pourrait revigorer les liens entre la philosophie politique et l'analyse littéraire/culturelle. Elle pourrait élargir les paramètres de la traduction pour englober une identité polyglotte dans les arts [...]. Le postcolonialisme pourrait promouvoir l'inclusion des Études Francophones dans une structure autre que celle d'un assimilationnisme "éclairé", menant ainsi à un plus grand intérêt pour les études françaises à l'étranger ». Par ailleurs, l'enseignement efficace des langues étrangères a un rôle-clé à jouer dans cela, dans la mesure où, sans la compétence nous permettant d'avoir accès à de nouvelles approches théoriques et de comprendre les événements sociopolitiques actuels, nous nous soumettons à une dépendance de la traduction.

Bien sûr, des progrès ont déjà été effectués dans cette direction, bien que les États-Unis soient derrière la Grande-Bretagne; par exemple, depuis quelque temps maintenant, les facultés de français embauchent des spécialistes de cinéma, de philosophie, d'études européennes, de moyens de communication et d'histoire (voir, par exemple, ce qu'offre le programme dans les universités d'Edinbourgh, de Bristol, de Nottingham, de Londres, de Leeds et Southampton). Tandis que l'expression d'une certaine ouverture à ces idées a été démontrée par le recrutement de professeurs dans ces domaines dans quelques facultés, et tandis que cela constitue, évidemment, un pas dans la bonne direction, ces mesures ne sont pas suffisantes à moins d'être accompagnées d'une véritable volonté et d'un engagement sincère à reconnaître que ces "réorientations " 
ne sont pas temporaires - reflétant une tendance populaire - mais plutôt des manières plus permanentes de "repenser ", « rajeunir » et "réaligner " l'orientation des programmes universitaires. Paradoxalement, plutôt que de menacer l'alignement disciplinaire, ces nouveaux paradigmes devraient être adoptés comme garants des études françaises en tant que domaine. L'objectif ne devrait pas être l'hospitalité ou la cohabitation, mais la formulation de philosophies pédagogiques qui reflètent les origines coloniales étroitement imbriquées dans ces domaines et leur potentiel continu pour la pollinisation croisée. Certes, l'interdisciplinarité elle-même doit éviter à tout prix les écueils inhérents à toute sorte d'institutionnalisation, condition pré-requise pour la protection et la garantie des exigences revitalisantes et dynamiques qui maintiennent les disciplines en vie.

Les enseignants ont la responsabilité de concevoir des programmes innovants regroupant plusieurs siècles et domaines de spécialisation et de formation qui soulignent la relation entre les études françaises et francophones, sondant les liens entre : le surréaliste André Breton et le poète martiniquais Aimé Césaire, les préfaces de Jean-Paul Sartre aux ouvrages de Léopold Sédar Senghor (Sartre 1948), Frantz Fanon (ibid. 1961) et Albert Memmi (ibid. 1973); Jean-Loup Amselle (2001) au sujet du multiculturalisme ou Michel Wieviorka (2009) sur la diversité ; les perspectives croisées sur la «fracture coloniale» et les « ruptures postcoloniales» (Blanchard et al. 2005; Bancel et al. 2010); les "enjeux politiques de l'histoire coloniale", les identités diasporiques, ainsi que la "République coloniale» ou la "République impériale » (Coquery-Vidrovitch 2009; Tshimanga et al. 2009; Bancel et al. 2003; Le Cour Grandmaison 2009); la francophonie et la globalisation aujourd'hui; bref, les possibilités et les configurations sont infinies. Les étudiants de troisième cycle ont l'opportunité d'explorer ces nouveaux alignements au-delà des frontières délimitées entre les sciences humaines et sociales, mais également à travers les structures transcoloniales et transnationales. Notre tâche consistera à les aider à innover, tracer de nouvelles routes et délimiter de nouveaux sentiers critiques pour le futur. Avec une énergie, un enthousiasme, une imagination et une inventivité illimités, ce sont eux qui fourniront les coordonnées du chemin à venir. Leurs projets exploreront peut-être les ouvrages de Hanif Kureishi et Azouz Begag, Zadie Smith et Calixthe Beyala, Monica Ali et Faïza Guène, Abdourahman Wabéri et Nuruddin Farah, Ousmane Sembène et Ngùgì wa Thiong'o, mais aussi ceux de théoriciens tels que Bhabha et Mbembe. Nous devrions encourager les étudiants à mener des recherches innovatrices sans se sentir obligés de formuler des projets de thèse qui s'intègrent dans des boîtes clairement compartimentées, pour la simple raison que les facultés ne devraient pas ressembler demain à ce à quoi elles ressemblent aujourd'hui.

\section{BIBLIOGRAPHIE}

AMSELLE, J.-L., 2001 Vers un multiculturalisme français. L'empire de la coutume, Paris, Flammarion. AMUTA, C., 1989 The Theory of African Literature: Implications for Practical Criticism, London, Zed. 
APPIAH, K. A., 1992 « The Postcolonial and the Postmodern », in K. A. APPIAH, My Father's House : Africa in the Philosophy of Culture, New York, Oxford University Press : 137-157.

APTER, E., 1995 « French Colonial Studies and Postcolonial Theory », Sub-Stance, 24 (76-77) : 169-180.

ASHCROFT, B., 2001 Post-Colonial Transformations, London, Routledge.

ASHCROfT, B., GRIFFITHS, G. \& TIFfin, H. (eds.), 1995 The Post-Colonial Studies Reader, London, Routledge.

BANCEL, N., BLANCHARD, P. \& VERGÈs, F., 2003 La République coloniale : essai sur une utopie, Paris, Albin Michel.

BANCEL, N., BERNAULT, F. \& BLANCHARD, P. (dir.), 2010 Ruptures postcoloniales : les nouveaux visages de la culture française, Paris, La Découverte.

BARNABÉ, J., CONFIANT, R. \& CHAMOISEAU, P., 1989 Éloge de la créolité, Paris, Gallimard.

BEHR, M., 2000 Embrace, London, Little.

BLANCHARD, P., BANCEL, N. \& LEMAIRE, S. (dir.), 2005 La fracture coloniale : la société française au prisme de l'héritage colonial, Paris, La Découverte.

CÉSAIRE, A., 1955 Discours sur le colonialisme, Paris, Présence Africaine.

CHAKRABARTY, D., 2009 Provincialiser l'Europe : la pensée postcoloniale et la différence historique, Paris, Éditions Amsterdam.

CHINWEIzu, O. J., 1980 Toward the Decolonization of African Literature, Enugu (Nigeria), Fourth Dimension.

COHEN, B., 1984 The French Encounter with Africans, Bloomington, Indiana University Press.

CONKLIN, A. L., 1997 A Mission to Civilize : The Republican Ideal of Empire in France and West Africa, 1895-1930, Stanford (Ca.), Stanford University Press.

COQUERY-VIDROVITCH, C., 2009 Enjeux politiques de l'histoire coloniale, Marseille, Agone.

DIOP, C. A., 1954 Nations nègres et cultures, Paris, Présence Africaine.

-, 1960a L’Afrique noire précoloniale, Paris, Présence Africaine.

-, 1960b L'Unité culturelle de l'Afrique noire, Paris, Présence Africaine.

-,1960c Antériorité des civilisations nègres, Paris, Présence Africaine.

-, 1981 Civilisation ou Barbarie, Paris, Présence Africaine.

DIOP, D. M., 1992 Hyènes, 110', Sénégal, Kino International.

ELLIS, G., 1994 The Drilling Fields, 50', United Kingdom Catma Films.

FANON, F., 1952 Peau noire, Masques blancs, Paris, Éditions du Seuil.

GANDHI, L., 1998 Postcolonial Theory : A Critical Introduction, New York, Columbia University Press.

GLISSANT, É., 1981 Discours caribéen, Paris, Éditions du Seuil.

GUGELBERGER, G. (ed.), 1985 Marxism and African Literature, London, James Currey.

KRITZMAN, L., 1995 «Identity Crises : France, Culture and the Idea of the Nation », Sub-Stance, 24

$(76-77): 5-20$. 
LACAPRA, D., 2000 « Reconfiguring French Studies », in D. LACAPRA (ed.), History and Reading: Tocqueville, Foucault, French Studies, Toronto, University of Toronto Press : 199-226.

LE COUR GRANDMAISON, O., 2009 La République impériale. Politique et racisme d'État, Paris, Fayard. LIONNET, F., 1995 Postcolonial Representation : Women, Literature, Identity, New York, Cornell University Press.

MBEMBE, A., 1999 « Les frontières mouvantes du continent africain », Le Monde Diplomatique (novembre) : 22 .

-, 2000 De la Postcolonie : essai sur l'imagination politique dans l'Afrique contemporaine, Paris, Karthala. MCCLINTOCK, A., MUFTI, A. \& SHOHAT, E. (eds.), 1997 Dangerous Liaisons : Gender, Nation, and Postcolonial Perspectives, Minneapolis, University of Minnesota Press.

MILLER, C. L., 1985 Blank Darkness : Africanist Discourse in French, Chicago, Chicago University Press. -, 1990 Theories of Africans : Francophone African Literature and Anthropology, Chicago, Chicago University Press.

MOORE-GILBERT, B., 1997 Postcolonial Theory : Contexts, Practices, Politics, London, Verso.

NORA, P., 1984-1992 Les lieux de mémoire, Paris, Gallimard.

ORISHA, I., 1986 « Sony Labou Tansi face à douze mots », L'Équateur, 1 : 30.

SARTRE, J.-P., 1948 « Orphée Noir », préface à L.-S. SENGHOR, Anthologie de la nouvelle poésie nègre et malgache de langue française, Paris, Presses universitaires de France.

-, 1961 Préface à F. FANON, Les damnés de la terre, Paris, Maspero.

-, 1973 Préface à A. MEMMI, Portrait du colonisé précédé de Portrait du colonisateur, Paris, Payot.

SENGHOR, L.-S., 1964 Liberté I : Négritude et humanisme, Paris, Éditions du Seuil.

-, 1971 Liberté II : Nation et voie africaine du socialisme, Paris, Éditions du Seuil.

-, 1977 Liberté III : Négritude et civilisation de l'universel, Paris, Éditions du Seuil.

-, 1983 Liberté IV : Socialisme et planification, Paris, Éditions du Seuil.

SMITH, Z., 2001 White Teeth, New York, Vintage.

,- 2003 Sourires de loup, Paris, Gallimard.

THOMAS, D., 2002 Nation-Building, Propaganda, and Literature in Francophone, Bloomington, Indiana University Press.

-, 2007 Black France : Colonialism, Immigration and Transnationalism, Bloomington, Indiana University Press.

TSHIMANGA, C., GONDOLA, D. \& BLOOM, P. J. (eds.), 2009 Frenchness and the African Diaspora: Identity and Uprising in Contemporary France, Bloomington, Indiana University Press.

WIEVIORKA, M., 2009 La diversité : rapport à la ministre de l'Enseignement supérieur et de la Recherche, Paris, Robert Laffont.

WILliAMS, P. \& CHRISMAN, L. (eds.), 1994 Colonial Discourse and Post-Colonial Theory : A Reader, New York, Columbia University Press. 


\section{NOTES}

2. Pour une perspective historique sur ces questions, consultez les ouvrages de William B. COHEN (1984) et d'Alice L. CONKLIN (1997).

3. Voir aussi Christopher milleR (1990 : 24), Anthony APPIAH (1992) et Jemie CHINWEIZU (1980 : 141).

4. Voir aussi Dipesh CHAKRABARTY (2009).

\section{RÉSUMÉS}

Résumé

L'objectif que nous proposons consistera à interroger l'histoire longue et compliquée de la France avec l'Afrique, de manière à mettre en relief les composantes et les contributions africaines aux études francophones, à souligner l'importance des modèles théoriques africains et à explorer et remettre en question la manière dont les études francophones et postcoloniales ont partagé, contesté, et même joui d'un terrain discursif de proximité sous l'égide d'une vaste gamme d'alignements disciplinaires aux États-Unis. Ce cadre nous permettra de retracer les nouvelles coordonnées et trajectoires intellectuelles de ces domaines et d'évaluer leur utilité et leur pertinence dans le processus de déballage des divers phénomènes culturels.

Abstract

Our purpose is to query France's long and complex relationship with Africa, and to highlight the African components and contributions to Francophone studies. We will stress the importance of African theoretical models and explore (as well as challenge) the way French and postcolonial research has shared, contested, and even enjoyed, a close discursive terrain in the United States under the aegis of a vast range of disciplinary combinations. This framework will enable us to describe new orders and intellectual trajectories in these areas and evaluate their use and relevance to the process of unravelling a number of cultural issues.

\section{INDEX}

Mots-clés : diaporas africaines, études francophones, francophonie, histoire coloniale, théorie postcoloniale

Keywords : African Diasporas, Francophone Studies, French Speaking World, Colonial History, Postcolonial Theory

\section{AUTEUR}

\section{DOMINIC THOMAS}

Département d'études françaises et francophones, Université de Californie, Los Angeles (UCLA). 